\title{
A Testbed System for Robotically Assisted Percutaneous Pattern Therapy
}

\author{
Andrew Bzostek ${ }^{1}$, Aaron C. Barnes ${ }^{2}$, Rajesh Kumar ${ }^{1}$, James H. Anderson ${ }^{3}$, \\ and Russell H. Taylor ${ }^{1}$ \\ ${ }^{1}$ Department of Computer Science, The Johns Hopkins University \\ ${ }^{2}$ Department of Mechanical Engineering, The Johns Hopkins University \\ ${ }^{3}$ Department of Radiology, The Johns Hopkins University
}

\begin{abstract}
This paper presents a second generation prototype image-guided robotic system for percutaneous delivery of surgical devices and therapeutic agents, with potential applications in the treatment of liver cancer and other malignancies. The system is intended to deliver these devices and therapies more consistently and accurately than a physician can achieve freehand. This capability will permit the treatment of smaller lesions, will enable the physician to better achieve optimized patterns of therapy distribution, and will allow for more rapid re-targeting for multiple lesions. The system will allow treatment of patients for whom surgery is contraindicated and could potentially replace major surgery, reducing patient morbidity and mortality, as well as the cost of treatment. The new prototype system uses new, modular hardware and software components, which improve its accuracy, usability, and flexibility as compared to the first generation prototype. Techniques for image processing, both in $2 \mathrm{D}$ and 3D images, planning, and fiducial registration have also been developed. In vitro, the system achieves submillimetric accuracy in the placement of simulated treatment devices from a preoperative plan generated from 3D imagery. Some initial in vivo considerations have also been addressed, and work is ongoing in this area.
\end{abstract}

\section{Introduction}

Providing therapy in a minimally invasive manner can afford significant improvement to traditional surgery for a variety of procedures, and in many cases such techniques are already accepted as the preferred form of treatment.

With an estimated 500,000 to 1 million new hepatocellular carcinoma (HCC) cases annually, liver cancer is, globally, one of the most common malignancies [1]. Though approximately 16 thousand new cases of liver and biliary passage cancer are diagnosed each year in the US [2], with increasing hepatitis incidence in this country, the incidence of HCC is expected to increase significantly.

Developing minimally invasive treatments for liver malignancies has attracted significant interest. Such treatments seek both to reduce the invasiveness associated with lesion resection, and to provide treatment in cases for which surgical resection is not indicated. Surgical resection is associated with some patient morbidity and mortality, but it remains the standard for treatment of both primary and metastatic 
hepatic tumors. However, even for patients for whom disease is confined to the liver, surgery is often not an option, because of an inoperable lesion location, associated comorbid conditions, or the lack of sufficient hepatic parenchyma reserve to tolerate a major resection.

Percutaneous treatment modalities offer local control and potential prolongation in survival for patients with liver tumors, while reducing the risk associated with standard liver resection [3]. Methods under investigation include ethanol injections [4], cryotherapy [5], radiofrequency ablation [6,7], interstitial radiation [8], and laser hyperthermia [9]. Using real-time ultrasound or fluoroscopy for guidance, these therapies are typically delivered freehand. It is often difficult to approach small lesions $(<2 \mathrm{~cm})$, specific tumor regions are difficult to target, and larger lesions often require multiple applications to a given tumor. The clinical value of these therapeutic approaches could be significantly improved with the ability to deliver these therapies more precisely in a planned pattern.

We are currently developing a system that will assist in the percutaneous placement of treatment patterns $[10,11,12,13,14]$. The system utilizes preoperative 3D imaging (CT or MRI) for planning and uses intraoperative, real-time fluoroscopy to guide a robotic system to align and deliver therapy to target locations. The ability to consistently place a therapy pattern with high accuracy will not only improve currently available treatments, but will also foster the development of new therapies which rely on it.

This paper presents this system and preclinical validation results. The rest of the introduction will discuss previous work and our target clinical procedure. In Section 2, we will present the hardware and software components of the new prototype system. Section 3 presents the results from a series of validation experiments, including an in vitro, end-to-end assessment. The paper will conclude with a discussion of the implications of these results as well as our path for future work.

\subsection{Previous Work}

There has been some previous work dedicated to image-guided needle placement in soft tissue. Some of these systems rely on the fixed relationship between the treatment delivery system and the image, e.g. [15], and some, e.g. [16,17] use only a passive positioning aid. One previous system, the Picker PinPoint does address problem of pattern placement, but this system uses a passive manipulator fixed in a known way to a single, proprietary imaging system. We believe that use of an active positioning device imparts significant benefits, including higher accuracy, repeatability and speed of alignment, that justify a more complex system. Additionally, not having to rely upon a priori knowledge of imager to positioning device fixation makes the system significantly more flexible, both in terms of reachable anatomical targets, and the choice of guidance imaging modality.

\subsection{The Target Procedure}

We are investigating a broad range of percutaneous scenarios, but in order to focus our discussion, have defined a reference procedure, which has been described more fully in [11]. For the discussion here, suffice it to say that fiducials, hand implanted 
prior to 3D planning imagery, serve as guides for target registration under intraoperative, biplanar fluoroscopy. Also, for each planned treatment insertion, reregistration, alignment, needle insertion, treatment deposition, and needle removal must be completed within a single breath-hold $(\sim 20 \mathrm{sec})$

\section{Methods}

\subsection{Hardware}

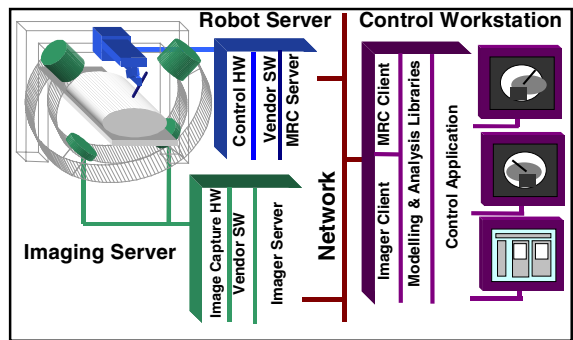

Fig. 1. System Architecture

As discussed in previous papers [10,11], previous tests were conducted using a LARS surgical robot developed at IBM research and a treatment insertion device.

The current prototype system uses an XYZ translation platform and a simulated treatment injector from the Hopkins Modular Robotic Family. The current robot is positioned via a cart-mounted U-frame and 3 passive, lockable degrees of freedom. This configuration allows it to reach into the field of view (FOV) of the intraoperative imaging system without impeding patient positioning, restricting its work volume or interfering with the collected images.

We have constructed a second-generation injector for our automatic injection system described in [11]. This injector is designed to overcome the first-generation injector's shortcomings, including a relatively violent release mechanism and asymmetric intravenous point needle, both of which contributed to significant simulated therapy seed shift upon release. The improved injector utilizes a computer controlled release mechanism. This release mechanism allows adjustment for varying therapy size and verification of therapy release. The simulated therapy consists of a 0.5 " length of 13-gauge stainless steel hypodermic tubing. held via a diamond tipped trocar matched to the inner diameter of the tubing. The therapy rod is released from the injector by withdrawing the trocar from within the tubing.

Intraoperative computational capabilities are provided by a group of workstations connected by a local area network. Currently, only two workstations are used, one of which provides robot control and the other which both captures images and provides a user interface and application level control.

\subsection{Software}

The new prototype robotic system is controlled through the Modular Robot Control (MRC) Library developed at JHU, which provides Cartesian level control. Image capture and processing are done via a image control library written on top of Matrox Imaging's MIL library

The basic imager calibration and robot registration techniques used have been presented elsewhere [11]. In previous experiments, a mean image-guided placement accuracy of $0.43 \mathrm{~mm}$ [14] and intra-operative plan-based pattern placement with an 
accuracy of $0.52 \mathrm{~mm}$ [11]. In order to allow the system to accurately create, register, and execute planned patterns created from preoperative 3D imaging, several new algorithms have been developed.

Bead Image Processing. Fundamental to the accuracy of the system is its ability to find the centers of spherical fiducial markers, both in tomographic and projective imaging. In order to maximize the system's accuracy, we have developed two novel techniques which improve the accuracy of these measurements.

Projective Fluoroscopic Imagery. The most basic technique for finding the projection of the center of a bead in a fluoroscopic images is weighted centroid determination. Two problems arise, though, when the bead of interest is projected over a region of non-uniform density. First, it can be quite difficult to identify seeds automatically, second (see Figure 2) such a region will skew the determination of the center downward along the gray level gradient. For small, dense spheres, this is only a small problem, however, when using the larger, less radioopaque markers, the calculated

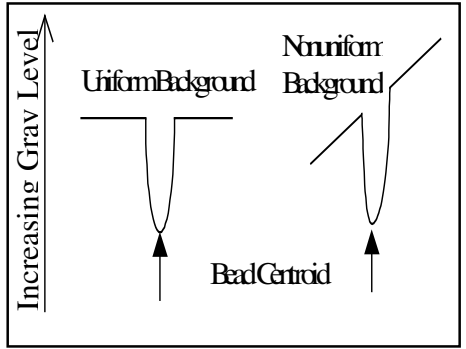

Fig. 2. The effect of a background gradient on bead centroid determination centers are often skewed by several pixels. In order to remove this affect, we have developed a novel technique, which processes a single image to remove most of the background influence.

Given a log image of a fiducial, our technique uses pixels in an annular neighborhood at a distance $r$ from the pixel of interest to calculate the direction of the gradient, then corrects the pixel's value by subtracting the average value of pixels at distance $r$ in the direction perpendicular to the calculated gradient. The technique can be applied efficiently, for small $r$, over entire images, and can thus be used prior to any bead localization. The first step is to calculate the estimated gradient vector $\mathbf{V}$ :

$$
\mathbf{V}=\sum_{\mathbf{d} \in S}\left[[I(\mathbf{t}+\mathbf{d})-I(\mathbf{t}-\mathbf{d})] \frac{\mathbf{d}}{\|\mathbf{d}\|}\right]
$$

where $\mathbf{t}$ is the target pixel, $\mathbf{d}$ is a displacement selected from the set $S$ of all integral vectors in the annular neighborhood and $I(\mathbf{p})$ is the image intensity at position $\mathbf{p}$. Once $\mathbf{V}$ is calculated, calculating $I^{\prime}(\mathbf{t})$, the corrected intensity, is simply:

$$
I^{\prime}(\mathbf{t})=I(\mathbf{t})-\frac{I\left(\mathbf{t}-r \hat{\mathbf{V}}_{\perp}\right)+I\left(\mathbf{t}+r \hat{\mathbf{V}}_{\perp}\right)}{2}
$$

There $r$ is the average annular radius, $\hat{\mathbf{V}}_{\perp}$ is the unit vector in the direction
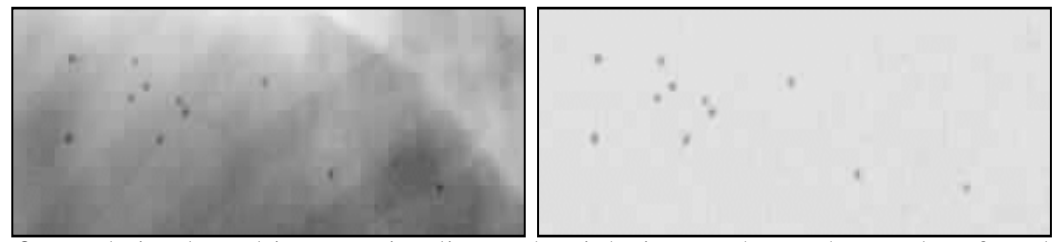

Fig. 3. Beads implanted in a porcine liver. The right image shows the results of applying the linear gradient correction with a radius of 3 pixels followed by a $3 \times 3$ gaussian smoothing operation to the left image, a raw fluoroscopic image after a log correction to linearize its density response. 
perpendicular to $\mathbf{V}$, and $I$ of a non-integral position is the linear interpolation from the position's four integral neighbors.

This correction is calculated identically for all pixels within the region of interest, thus $r$ is set to twice the radius of the beads we are interested in finding. This technique not only allows for the accurate calculation of projected bead centers [see section 3 for results], but is also quite useful for separating out beads in a complex image (see fig. 3).

Volumetric Images. Our system also relies upon the accurate localization of the fiducial beads in the 3D preoperative planning image. This is particularly important in the interslice direction $(\mathrm{Z})$, where the resolution can be as small as $1 / 10$ that in the two intraslice directions When faced with similar problems, Hanson, et al [18] and Lewis et al[19,20] chose to use fiducials which are large and implanted in bone, while Ellis et al[21] utilizes details of the slice geometry in CT scanners to localize small, very dense tantalum spheres, which, while nicely visible on fluoroscopic imagery, cause artifacts in CT.

Currently, we are working with $1 / 8$ " Aluminum Oxide fiducials. The material was chosen, both for its biocompatibility and its balance of contrast in fluoroscopy and lack of artifacts in CT. Unfortunately, many clinical CT scans have an interslice distance of $3 \mathrm{~mm}$, just smaller than the bead diameter. This leads to a inaccuracies when using the standard weighted centroid calculation for determining the bead center in the $\mathrm{Z}$ direction. To solve this problem, we have derived a method which uses the known geometry of the fiducial to allow us to much more accurately find its center in this direction.

Given a sphere of radius $r$ centered at the origin, the area $A$ of a slice at signed distance $|d|<=r$ from the center is given by: $A=\pi s^{2}=\pi\left(r^{2}-d^{2}\right)$, (figure 4) where $s$ is the radius of the circular slice. Such a slice divides the sphere into two regions. Without loss of generality, we can assume that the normal to the slice is along the $z$ direction and that it is displaced in the positive $z$ direction. Thus the volume of smaller of the two regions is:

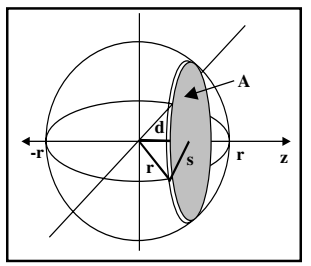

Fig 4. Slicing a sphere

$$
\begin{aligned}
V_{1} & =\int_{d}^{r} A(z) d z \\
& =\frac{2}{3} \pi r^{3}-\pi d\left[r^{2}-d^{2} / 3\right]
\end{aligned}
$$

and the volume of the larger region is $V_{2}=\frac{2}{3} \pi r^{3}+\pi d\left[r^{2}-d^{2} / 3\right]$. The sum and difference are:

$$
\begin{aligned}
V_{\text {SUM }} & =\frac{4}{3} \pi r^{3} \\
V_{\text {DIFF }} & =2 \pi d\left[r^{2}-d^{2} / 3\right]
\end{aligned}
$$

For a sphere with uniform density $\delta$, these can be converted to masses simply by multiplying each by this $\delta$. This factor, though, can be removed by taking the ratio of the two values:

$$
R_{V}=\frac{V_{D I F F}}{V_{S U M}}=\frac{3}{2}\left[\frac{d}{r}-\frac{1}{3} \frac{d^{3}}{r^{3}}\right]
$$


Given a known radius and a measurement of the mass on each side of the cut, we would like to solve for the offset of this cut. Solving for a cubic equation in $d$, we get:

$$
d^{3}-3 r^{2} d+2 r^{3} R_{V}=0
$$

where $r$ is known and $R_{v}$ is measured. Since the zeros of the derivative with respect to $d, 3 d^{2}-3 r^{2}$, occur when $|d|=r$, and we've assumed that $|d|<=r$, we only need to solve for the central root, which will be real. In fact, since this region of the equation is monotonic, we can use a binary search to find an arbitrarily accurate estimate for $d$ in time proportional to the log of the inverse of the size of the desired maximum error.

In a tomographic image set where the slice spacing is equal to the slice thickness, we can take the two sliced volumes as the portions of the sphere on either side of a voxel (slice in the $\mathrm{Z}$ direction) boundary, and $d$ is the boundary's distance to the sphere's center. In order to find a value proportional to the total mass on each side, the partial mass of sphere within each voxel is esimated using an approximate density value for the background, which can be estimated for the neighborhood of the sphere. Once $d$ is calculated, the sphere's center position in the direction perpendicular to the cut is simply the cut's position plus $d$. This technique shows promise for the precise, slice spacing-independent calculation of the fiducials' centers. (see section 3.4 for results)

Planning. We have developed a system for planning the arbitrary path brachytherpies that our prototype can implement. This system uses user input to select an approach direction, then projects the problem into $2 \mathrm{D}$, where it is solved using a modified gradient descent method. The target points are then projected back into lines, whose depth and length are calculated to cover the target. While this is only an initial prototype planner and still requires significant verification, it provides a useful platform for testing the rest of the system.

Pattern Registration. Given 3D positions for fiducials in two spaces, we now need to: 1 . find the correct pairing of these positions, and 2, find the best fit transformation between them. Unfortunately, the intraoperative set may either be incomplete-containing points not matched in the preoperative one, or may have deformed. In order to make the specified problem solvable, we will assume that there is a known upper bound on the displacement the non-rigid deformation causes from the best fit rigidly transformed location, and that the fiducials are at least twice this far apart. These assumptions are not unreasonable, given the spacing of the fiducials $(\sim 1-2 \mathrm{~cm})$ as compared to the level of non-rigid motion we've observed in fiducials within the same lobe of a porcine liver (up to $\sim 3 \mathrm{~mm}$ ).

Our algorithm iteratively solves for both the matching and transformation, first matching an arbitrary triple of points, then iteratively pairing the remaining best fit pairs and updating the transformation. If the matching is unsatisfactory, then a different initial set of three beads from set 1 are chosen and the procedure repeated. If $m$, the number unmatched beads in set 1 is less than $1 / 3$ the number of beads in the set, this will take at most $m$ attempts. Each application of this algorithm takes, worst case, time proportional to $n^{5}$, though in practice it is generally much smaller because 
there tend to be very few candidate matches for the $2^{\text {nd }}$ and $3^{\text {rd }}$ points in the initial pairing, given a match for the $1^{\text {st }}$.

Once the matching and best-fit transformation has been established, small perturbations in the intraoperative fiducial positions are easily handled. This process has been automated and takes less than a second on inexpensive computational hardware.

Phantom Construction. We constructed in vitro phantoms for each of our experiments. These phantoms consisted approximately cylindrical plastic containers, approximately 4 inches in diameter, and approximately 4 inches tall. These containers were filled with agarose gel at $1.3 \%$ concentration. Tumor phantoms were created by creating a smaller cylinder of $1.3 \%$ agarose with a suspended Barium solution. Fiducials were $1 / 8$ " radius Aluminum Oxide spheres.

Table 1. Results from in-vivo bead motion study

\section{Experimental Results}

\begin{tabular}{|l|l|r|r|r|}
\hline Comparison & Condition & \multicolumn{1}{c|}{$\begin{array}{c}\text { Avg Error } \\
(\text { beads })\end{array}$} & $\begin{array}{c}\text { Max Error } \\
(\mathrm{mm})\end{array}$ \\
\hline & & 336 & 0.75 & 1.59 \\
\hline Within Sessions & Different Poses & 100 & 0.40 & 0.87 \\
& Same Pose & 40 & 1.11 & 2.12 \\
\hline \multirow{2}{*}{ Between Sessions } & Different Poses & 7 & 1.15 & 2.18 \\
\hline & Same Pose & &
\end{tabular}

\subsection{Bead Stability/ Deformation}

Bead stability and non-rigid deformation are a concern, so we have conducted studies to gain a better appreciation of the motion which in vivo implanted beads will undergo. Two in-vivo experiments were conducted in which a set of beads were implanted manually and imaged in several poses, in two sessions separated by several days. The results in table 1 represent residual errors in bead location prediction after using a thin-plate spline based on a sub-set of the fiducials to correct for their deformation. Table 1 summarizes the results from the first study.

The second study yielded some interesting, if less quantitative results. For this study, a small set of beads were implanted within a lobe different from the primary insertion site, and within the primary lobe, some fiducials were implanted more superficially than others. Insufficient beads were available to do a good TPS correction, but within a group of fiducials implanted within the interior of the primary lobe, deformative motion was small (approx. $1 \mathrm{~mm}$ without correction). Beads implanted more superficially showed more significant deformation (approx. $3 \mathrm{~mm}$ max without correction), and beads implanted in different lobes showed quite significant deformation (5-10 $\mathrm{mm}$ max).

\subsection{Bead Localization in Fluoroscopic Images}

While our background subtraction has significantly improved the general automaticity of our application (and thus time to realign), we also wanted to quantify the improvement in bead center finding achieved by the algorithm. To do so, we imaged a phantom with 7 fiducials implanted. Their centers were determined using the 3D 
weighted-centroid method, from a $3 \mathrm{mmx} 3 \mathrm{~mm}$ CT scan, then from a pair of fluoroscopic images using weighted centroid, both before and after background correction w/ an $r=7$. The sub-pixel centroids differed by an average of 1.58 pixels $(\sim .3 \mathrm{~mm})$, though it should be noted that without background correction, care had to be taken to select seed points which actually corresponded to the centers of beads, while after background correction, these seeds could be determined semiautomatically. The 3D point sets were then registered to the set calculated from CT. The set without background correction showed a residual error of $1.68 \mathrm{~mm}$, while the set after correction showed a residual error of $1.2 \mathrm{~mm}$, demonstrating that most of the error represented by the difference in the image positioning occurred in the centers found prior to the background correction

\subsection{CT Plan Placement}

In order to quantify the end-to-end accuracy of our system, in vitro, we conducted a study in which a tumor phantom was suspended in undoped agarose gel. 7 fiducials were implanted by hand surrounding the tumor model A $3 \mathrm{mmx} 3 \mathrm{~mm}$ CT scan

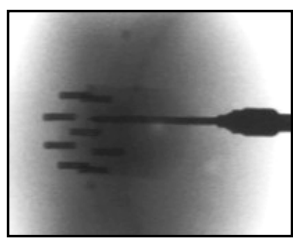

Fig. 6. Fluoroscopic image of implantation was collected, and a plan was created based on this scan. At that time, the fiducials were localized using only the standard weighted centroid method. The plan called for 9 rods to be inserted. After random initialization, the plan converged in 32 iterations to an approximately circular pattern, with one treatment placed at the center of the tumor. The experiment then moved to a biplanar fluoroscopic suite. After calibrating the imagers (avg residual error in fiducials $=0.49 \& 0.37$ pixels $)$ and registering the robot (placement error $(n=8)(m m)$ : mean $=0.314$, $\max =0.489$, std dev $=0.194)$, the phantom was placed in the working volume and imaged. The fiducials were isolated and a registration performed. [mean error $=1.35 \mathrm{~mm}]$. Using the robot, the simulated treatment rods were inserted. Between each insertion, the phantom was moved away from its starting position, replaced approximately, and the fiducial positions relocalized automatically [mean residual $=1.28 \mathrm{~mm}$, time $<1 \mathrm{sec}]$. After all of the rods were implanted, fluoroscopic images were taken to measure the position of the implanted rods. The fiducial locations in this image were registered to the CT-based positions (mean residual $=1.20 \mathrm{~mm}$ ), and this transformation applied to the bottom positions of the treatment rods. After removing known systematic displacements, their positions showed an average error of $0.765 \mathrm{~mm}$, $\max$ of $1.38 \mathrm{~mm}$, and a standard deviation of $0.339 \mathrm{~mm}$.

\subsection{CT Bead Localization}

To verify the accuracy of our 3D bead center calculation, a phantom containing 8 fiducials was created. Two $3 \mathrm{~mm} \times 3 \mathrm{~mm}$ CT scans of the phantom were
Table 2. RMS Error (mm) when registering fiducials. Comparison between techniques and images (WC $=$ Weighted Centroid, SS = Sliced-Sphere $)$

\begin{tabular}{l|l|l|l|l|l|}
\multicolumn{2}{c|}{} & \multicolumn{2}{|l|}{ Volume 1} & \multicolumn{2}{|l|}{ Volume 2} \\
\cline { 3 - 6 } \multicolumn{2}{c|}{} & WC & SS & WC & SS \\
\hline Volume 1 & WC & -- & 0.547 & $\mathbf{0 . 7 3 1}$ & 0.601 \\
\cline { 2 - 6 } & SS & 0.547 & -- & 0.600 & $\mathbf{0 . 2 7 1}$ \\
\hline Volume 2 & WC & $\mathbf{0 . 7 3 1}$ & 0.600 & -- & 0.560 \\
\cline { 2 - 6 } & SS & 0.601 & $\mathbf{0 . 2 7 0}$ & 0.560 & -- \\
\hline
\end{tabular}


collected, with the phantom rotated by approximately 90 degrees between scans. In each of the scans, the position of each of the fiducials was calculated using both a weighted centroid and our sliced-sphere technique. We then registered the point sets created by the different techniques within and between images (see table 2)

Our sliced-sphere technique shows almost a 3-fold improvement in RMS error when compared to the weighted centroid technique $(.27 \mathrm{~mm}$ vs $. .73 . \mathrm{mm})$ Even more importantly, it shows significantly less directional bias than those from the weighted centroid method, by comparing errors in the relative $\mathrm{Z}$ directions when registering point sets from both different images and different techniques $(.55 \mathrm{~mm}$ vs $.22 \mathrm{~mm})$.

\section{Discussion}

Though some work remains in validating the improved accuracy of the sliced-sphere fiducial localization technique, the system has demonstrated more than adequate baseline performance in vitro Many obstacles remain in the creation of a clinically viable system, including work on the injector end effector, planning system, and overall system robustness. From an engineering standpoint, however, the primary challenge that remains is to translate good in vitro performance into an in vivo setting. The primary obstacle to this goal is the deformation of the soft tissue targets. This deformation occurs both because of natural motion of the organ, and, intraoperatively, due to the insertion of the implantation needle. We have demonstrated that even a naïve non-rigid deformation model can account relatively well for the first source. Deformation due to needle insertion is a much greater challenge. We are currently pursuing work to use force measurements in conjunction with our imaging capabilities to characterize and hopefully, minimize and correct for, this kind of nonrigid motion.

\section{Conclusions}

In this paper, we have presented a prototype system for the precise and accurate percutaneous placement of patterns of therapy, with application to the treatment liver cancer as well as other malignancies. It uses an automated alignment and injection system which allows for the consistent, rapid alignment which is highly advantageous in this environment. We have presented image processing and fiducial registration techniques which have allowed us to build such a system. In particular, we have demonstrated a novel analytic geometry-based fiducial localization technique which offers almost a 3-fold improvement in RMS residual error when compared to the current standard. Most significantly, we have demonstrated a high level of end-to-end system accuracy in non-deforming in-vitro models, with a mean placement error of $0.75 \mathrm{~mm}$. 


\section{Acknowledgements}

The authors would like to acknowledge the work of Steve Schreiner in the development of the first generation prototype system. The research reported in this paper was supported in part by NSF/Whitaker Foundation for Cost Reducing Health Care Technology Grant \#5T32HL07712, in part by NSF Grant \#EEC9731478, in part by NSF equipment grant \#CDA-9529509, and in part by Johns Hopkins University internal funds.

\section{References}

1. J. R. Wands and H. E. Blum, "Primary Hepatocellular Carcinoma," The New England Journal of Medicine, vol. 325, pp. 729-731, 1991.

2. C. C. Boring, et al., Cancer Statistics, 1994," Cancer Journal for Clinicians, vol. 44, pp. 726,1994.

3. P. D. Schneider and J. P. McGahan, "Percutaneous Approaches to Liver Neoplasms," in Minimally Invasive Surgery, J. G. Hunter and 1. Sackier, Eds. New York: McGraw Hill, 1993, pp. 255-263.

4. T. Levraghi, A Salmi, and L Bolondi, 'Small hepatocellular carcinoma: percutaneous alcohol injection - results in 23 patients," Radiology, vol. 168, pp. 1313-1317,1988.

5. T. S. Ravikumar, "The Role of cryotherapy in the management of patients with liver tumors", Adv Surg 30:281-291, 1996.

6. A. E. Siperstein, S. J. Rogers, P. D. Hansen, A. Gitomirsky, "Laparoscopic thermal ablation of hepatic neuroendocrine tumor metastases," Surgery 122:6, 1146-1155, Dec 1997.

7. J. McGahan, P. Browning, and J. Brock, 'Hepatic ablation using radiofrequency electrocautery," Investigational Radiology, vol. 25, pp. 267-270,1990.

8. R. Holt, R. Naunta, and T. Lee, "Intraoperative interstitial radiation therapy for hepatic metastases from colorectal carcinomas," Amer. J. of surgery, vol. 54, pp. 231-233,1988.

9. J. Hahl, R. Haapiainen, and J. Ovaska, "Laser-induced hyperthermia in the treatment of liver tumors," Lasers Surg Med, vol. 10, pp. 319-321, 1990.

10. J. H. Anderson, et al., "Image-Guided Percutaneous Robotic Assisted Therapy,' presented at Annual Fall Meeting of the Biomedical Engineering Society, The Pennsylvania State University, 1996.

11. S. Schreiner, J. H. Anderson, R. H. Taylor, J. Funda, A. Bzostek, A. C. Barnes, "A System for Percutaneous Delivery of Treatment with a Fluoroscopically Guided Robot," Proceedings of the First Joint Conference of CVRMed-MRCAS, pp 747-756, Grenoble, France, 1997.

12. S. Schreiner, J. Funda, A. C. Barnes, and J. H. Anderson , "Accuracy Assessment of a Clinical Biplane Fluoroscope for Three-Dimensional Measurements and Targeting," presented at SPIE Conference on Medical Imaging, Newport Beach, CA, 1997.

13. A. Bzostek, S. Schreiner, A. C. Barnes, J. A. Cadeddu, W. W. Roberts, J. H. Anderson, R. H. Taylor, L. Kavoussi, "An Automated System for Precise Percutaneous Access of the Renal Collecting System", Proceeding of The First Joint Conference of CVRMed-MRC.AS, Grenoble, France, 1997.

14. S. Schreiner, et al., "Accuracy Assessment of a clinical biplane fluoroscope for threedimensional measurements and targeting," presented at SPIE Conference on Medical Imaging, Newport Beach, CA, 1997.

15. F. Burbank, S. H. Parker, "Stereotactic Core Breast Biopsy," Surgical Technology International II, pp. 179-186, 1993. 
16. P. Potamianos, B. L. Davies, and R. D. Hibberd, "Manipulator Assisted Renal Treatment," Proc. ARA/IFR Int. Conf. Robots for Competitive Industries, Brisbane, Australia, pp. 214227, 1993.

17. P. Potamianos, B.L Davies, and R.D. Hibbard, "Intra-operative Registration for Percutaneous Surgery," Proceedings of the 2nd Annual Symposium on MRCAS, Baltimore, MD, pp. $156-164,1995$.

18. W. H. Hanson, H. A. Paul, B. Williamson, and B.D. Mittelstadt, "Orthodoc: A computer system for presurgical planning." Proceedings of the $12^{\text {th }}$ IEEE Medicine and Biology Conference, 12: pp 1931-1932, Philadelphia, 1990.

19. J. T. Lewis, R. L. Lewis Jr., S. Schreiner, “An ultrasonic approach to localization of fidcial markers for interactive, image-guided neurosurgery part I: principles," IEEE Transoactions on Biomedical Engineering. v. 45 n. 5, pp 620-630, May 1998.

20. S. Schreiner, R. L. Galloway Jr., J. T. Lewis, : “An ultrasonic approach to localization of fidcial markers for interactive, image-guided neurosurgery part II: implementation and automation," IEEE Transoactions on Biomedical Engineering. v. 45 n. 5, pp 631-641, May 1998.

21. R. E. Ellis, S. Toksvig-Larsen, M. Marcacci, "Use of a Biocompatible fiducial marker in evaluating the accuracy of computer tomography image registration," Investigative Radiology, v. 31, n. 10, pp 658-667, Oct 1996. 